\title{
Asteroseismology and helium gradients in A-type stars
}

\author{
Sylvie Vauclair ${ }^{1}$ and Sylvie Théado ${ }^{2}$ \\ ${ }^{1}$ Laboratoire d'astrophysique de Toulouse, Observatoire Midi-Pyrénées, 14 avenue Edouard \\ Belin, 31400 Toulouse, France \\ email: sylvie.vauclair@obs-mip.fr \\ ${ }^{2}$ Institut d'Astronomie de Liège, Belgique \\ email: sylvie.theado @ulg.ac.be
}

\begin{abstract}
Asteroseismology may help detect diffusion-induced helium gradients inside MainSequence A stars. Models have been computed for 1.6 and $2.0 M_{\odot}$ stars with pure helium diffusion, at different ages, so that the helium gradient lies at different depths inside the star. The adiabatic oscillation frequencies have been analysed and compared with those of a model without diffusion. Clear signatures of the diffusion-induced helium gradient are found in the so-called "second differences". These frequency differences present modulations due to the partial reflection of the sound waves in the layer where the helium gradient occurs. A tentative application to the roAp star HD 60435 is presented.
\end{abstract}

Keywords. Asteroseismology; stars: abundances; stars: diffusion, stars: individual (HD 60435)

\section{Introduction}

It is now widely recognized that the abundance anomalies observed in peculiar A stars are basically due to element diffusion. Those for which the radiative acceleration is larger than gravity (like most of the metals) are pushed upwards while the other ones, like helium, diffuse downwards (Michaud 1970, Vauclair \& Vauclair 1982).

In this paper we show how asteroseismology can help detect helium gradients inside peculiar A stars. A more complete study is given in Vauclair \& Théado (2004). Rapid variations of the sound velocity inside a star lead to partial reflections of the sound waves, which may clearly appear as frequency modulations in the so-called "second differences" (Gough 1989):

$$
\delta_{2} \nu=\nu_{n+1}+\nu_{n-1}-2 \nu_{n}
$$

The presence of a diffusion-induced helium gradient leads to a kink in the sound velocity with a very clear signature in the oscillation frequencies. Asteroseismic observations of A-type stars can test for the presence of helium gradients.

In the present computations we neglected the effects of magnetic fields and mass loss (the stars were assumed to be spherically symetric) and we assumed that the modification of the stellar structure induced by metal accumulation in the outer layers was negligible. We computed the structural changes induced by helium settling and its influence on the oscillation frequencies. We applied this test to the only roAp star in which enough modes have been observed HD 60435 (Matthews et al. 1987).

\section{Seismic signatures of helium gradients}

Stellar acoustic $p$ modes with low $l$ values can propagate deeply inside the stars. For this reason, they may be used to obtain information on the deep stellar structure. In 

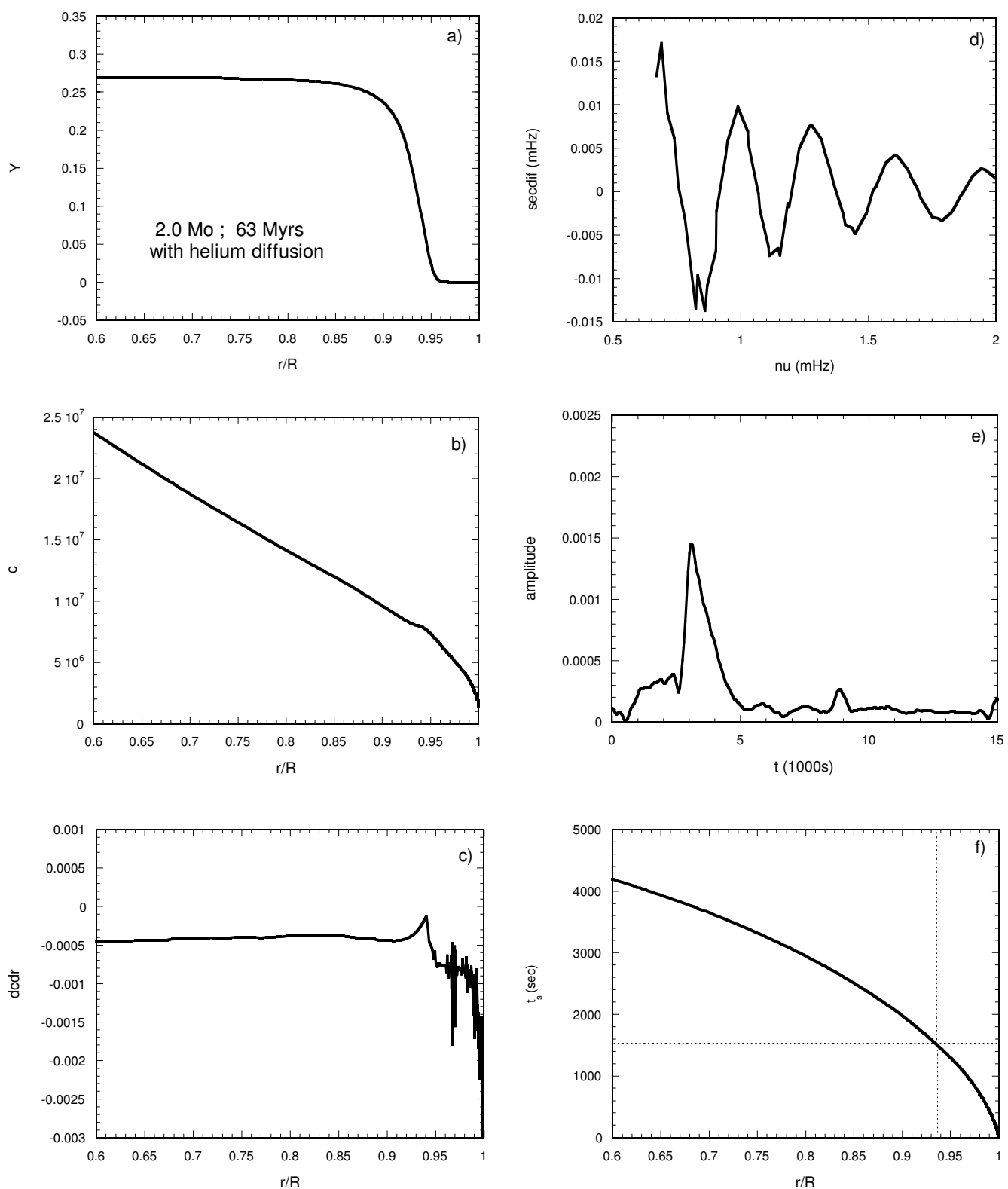

Figure 1. Helium diffusion and its consequences on the stellar structure and the oscillation frequencies for a $2.0 M_{\odot}$ at 63 Myrs, a) helium profile as a function of the fractional radius, b) sound velocity, a kink is clearly visible at the place of the helium gradient, c) first derivative of the sound velocity, the kink is still more visible, d) the second differences of the oscillation frequencies plotted as a function of the frequencies, e) the Fourier transform of graph (d) plotted as a function of time devided by one thousand (in seconds), f) the "acoustic depth", or time needed for the acoustic waves to travel from the surface to the considered radius, it can be checked that the peak of graph (e) corresponds to a time scale which is twice the "acoustic depth" of the helium gradient.

case of strong gradients in the sound velocity, which may be due to the boundary of a convective zone, to the helium ionization region or to helium gradients, acoustic waves are partially reflected. This creates modulations in the frequency values which are equal 
to $2 t_{s}$ where $t_{s}$ is the time needed for the acoustic waves to travel between the surface and the considered region (acoustic depth), i.e.,

$$
t_{s}=\int_{R}^{r} \frac{d r}{c}
$$
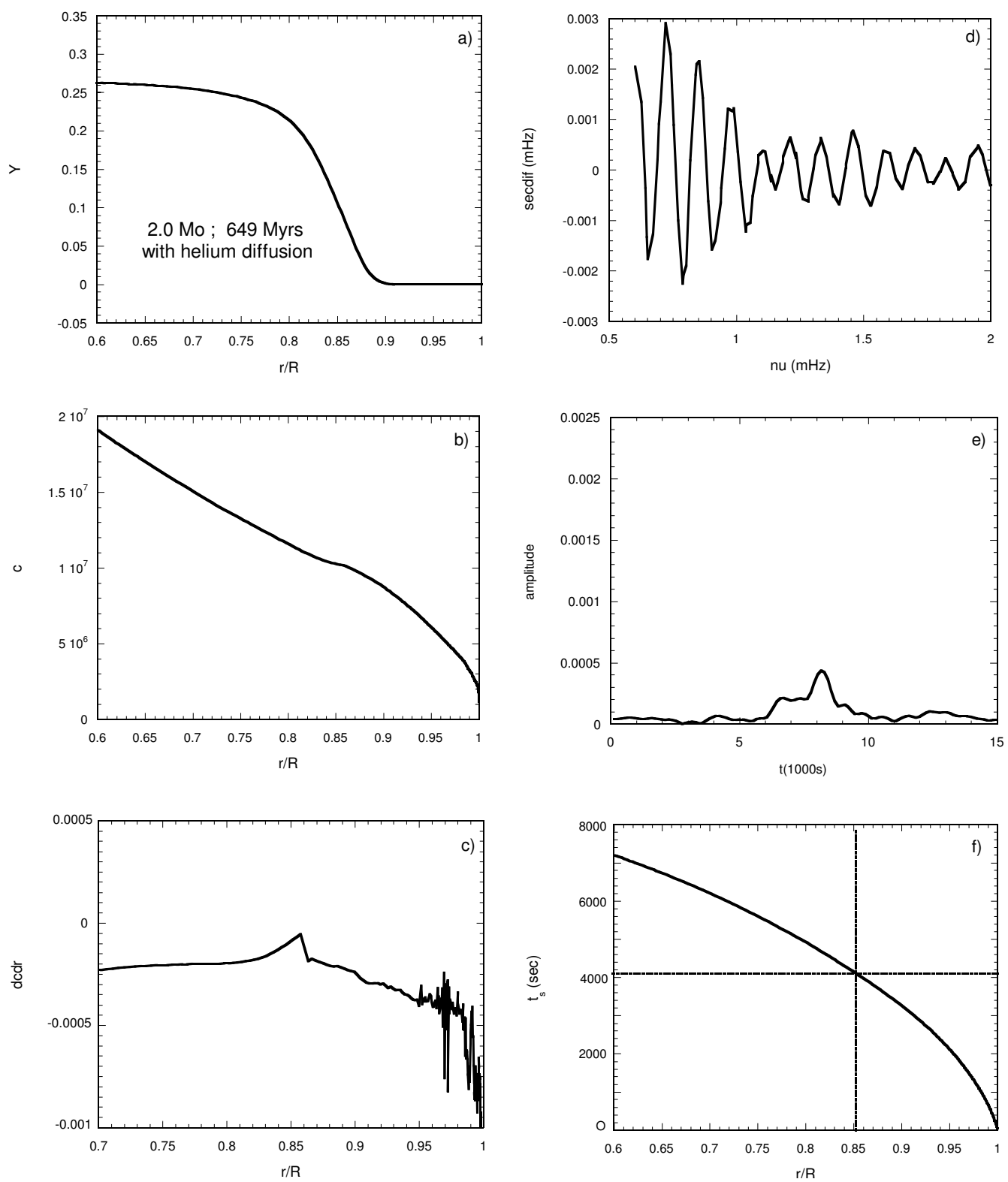

Figure 2. Same as Figure 1, for a $2.0 M_{\odot}$ model at 649 Myrs.

We studied the evolution of $1.6 M_{\odot}$ and $2.0 M_{\odot}$ stellar models, with pure helium settling. We have computed the oscillation frequencies and their second differences in five different models. Results are given in Figures 1 and 2 for the $2.0 M_{\odot}$ model with ages of 63 Myrs and 649 Myrs. In each figure, six graphs are displayed a) the helium profile 
inside the star, b) the sound velocity which shows a clear kink at the place of the helium gradient, c) the first derivative of the sound velocity in which the kink is still clearer, d) the second differences which show periodical oscillations, e) the Fourier transform of these oscillations, in which clear peaks are found for precise time values, and $f$ ) the time needed for the acoustic waves to travel between the surface and the considered radius, or "acoustic depth".

We can follow in these two figures the signatures of the helium gradients as they sink into the stars.

Similar computations have been done for evolution models in which helium diffusion is suppressed. The resulting second differences are quite different (Vauclair \& Théado 2004). They show signatures of the helium ionization zones while the signatures of helium gradients have, of course, disappeared as there is no helium left in the ionization regions.

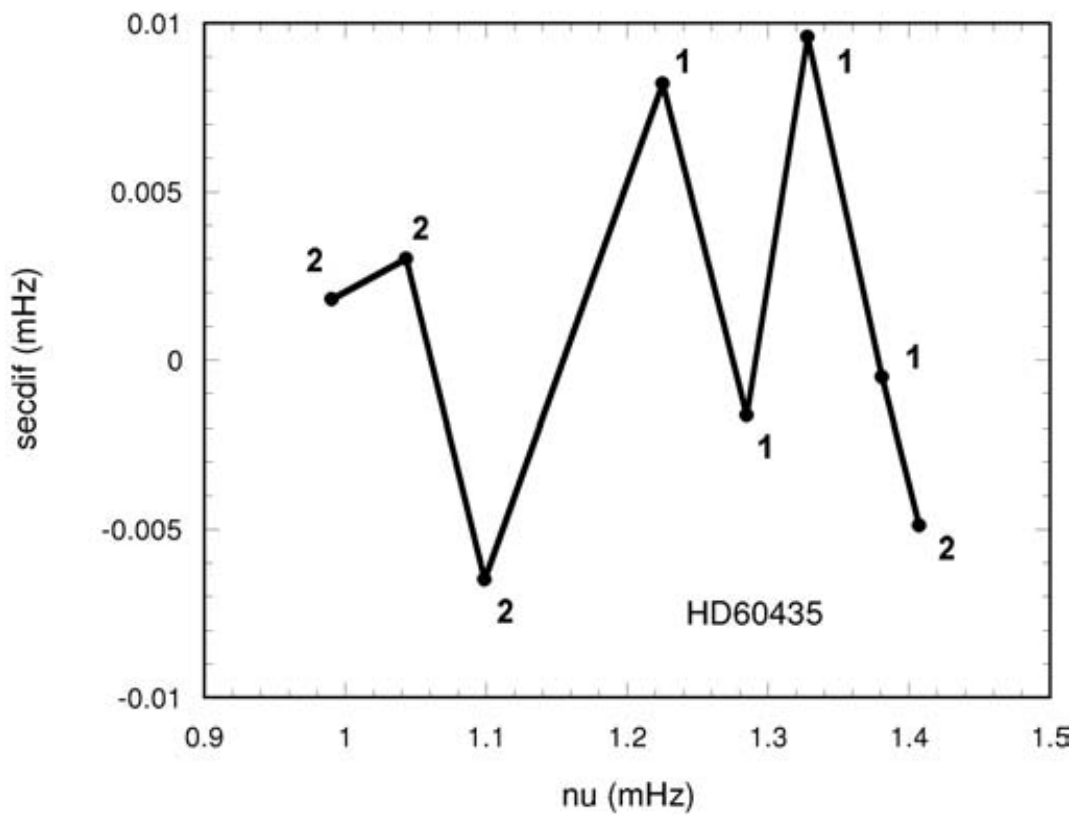

Figure 3. Second differences in the oscillation frequencies of HD 60435 as found from Matthews et al. (1987). The points are labelled according to their $l$ values.

\section{The roAp star HD 60435}

Among the rapidly oscillating Ap stars which have been detected up to now, only one, HD 60435, presents a frequency spectrum rich enough for the second differences to be computed (Kurtz, private communication). Matthews et al. (1987) discovered 17 frequency peaks in the Fourier spectrum, which they basically identified as $l=1$ and $l=2$ modes.

The computations of the second differences can only been done if enough modes with adjacent values of $n$ are detected for the same value of $l$. We have computed the observed second differences for HD 60435, using the frequencies and mode identifications as given by these authors, with an uncertainty of $\pm 0.0001 \mathrm{mHz}$. With the presently detected modes, eight second differences could be computed (Figure 3). They show a modulated trend similar to that of Figure 2, characteristic of the presence of a helium gradient below the surface (around $90 \%$ of the stellar radius). 
These results rely on the mode identifications which are given as "tentative" by Matthews et al. (1987). Note, however, that the difficulty in the identification by these authors was due to that the frequencies were not exactly at the expected value. This effect may simply be due to the presence of a helium gradient.

\section{Conclusion}

We have computed models of A-type Main Sequence stars with and without helium diffusion and we have shown that asteroseismology can give signatures of the presence of helium gradients below the surface. The analyses of the second differences in the oscillation frequencies show modulations which are due to the partial reflection of the acoustic waves at the place of the helium gradient.

At present these theoretical results can be compared to the observations of only one star, namely the roAp HD 60435. The computated second differences give evidence of a modulation similar to the one expected for the presence of a helium gradient below the surface.

Our theoretical computations have been done for spherically symetrical stars, without rotation induced mixing and ignoring any magnetic effects. We did not either include in the computations the abundance variations of heavy elements due to the combined effect of gravitational settling and radiative acceleration. This should be done in the future. These first results are, however, very encouraging and we hope that enough mode detections and identifications will be obtained in other stars to do similar tests.

\section{References}

Gough, D.O., 1989, in: Progress of Seismology of the Sun and Stars, proceedings of the Oji International Seminar Hakone, Japan, Springer Verlag, Lecture notes in Physics 367, p.283318

Matthews, J.M., Kurtz, D.W., Wehlau, W.H., 1987, Ap.J. 313, 782

Michaud, G., 1970, ApJ 160, 641

Vauclair, G., Vauclair, S., 1982, ARAEA 20, 37

Vauclair, S., Théado, S., 2004, A\&A 425, 179

\section{Discussion}

Cunha: Comment: Modes of different degree sample different latitudes of the star. For stars that are not speherically symmetric, that will probably prevent the surface effects from cancelling out in the second differences, as happens in spherically symmetric stars.

If in practice modes of different degree are used to calculate the observed 2 nd differences (rather than just modes of the same degree and orders), don't you think that it will introduce an uncertainity in your determination of depth of helium gradient?

VAUCLAIR: Thanks for mentioniong that. We should look more precisely at this question. This may be the reason why the observed peak (in the Fourier transform) is larger than the peaks computed in the spherically symmetric modes.

NoELS: I guess that in such a star, the excitation of modes is due to the H-ionization zone. Should not helium play a significant role?

VAUCLAIR: Yes, these results are consistent with the modes presented by Margarida Cunha where the excitation is due to $\mathrm{H}-\mathrm{K}$ mechanism, helium having diffused below. 
Grevesse: Your predictions disagree slightly with the observations but what is the accuracy of the obsevations?

VAUCLAIR: The relative uncertainities of the second differences are of order $10 \%$. A reasonable estimate of the uncertainities induced by the small number of observed frequencies lead to an uncertainity of $150 \mathrm{~s}$ on the acoustic depth of the helium gradient.

WeIss: Comment: The MOST will provide more examples for roAp's with a larger number of frequencies needed for your analysis. Question: What He-abundance should we use in our abundance analyses of roAP's? You showed that He can totally disappear in the atmosphere which would have a severe effect on model atmospheres to be used in abundance analyses.

VAUCLAIR: The computations have been done with pure microscopic diffusion. Turbulence could slow down the formation of helium gradient and let some helium in the atmosphere. In every case there should be an important helium depletion in the atnospheres of these stars, although I cannot tell at the present time how much would remain exactly. 